\title{
Importância e influência da estética dental relacionada à saúde biológica e social do indivíduo
}

Importance and influence of dental aesthetic related to the biological and social health of the individual Importancia e influencia de la estética dental relacionada con la salud biológica y social del individual Matheus Pedro Castro do Nascimento VIEIRA ${ }^{\mathbf{1}}$ José Henrique de Araújo $\mathbf{C R U Z}^{\mathbf{1}}$ Ruth de Souza MEDEIROS ${ }^{1}$

Abrahão Alves de OLIVEIRA FILHO²

Maria Angélica Satyro Gomes ALVES ${ }^{2}$

Camila Helena Machado da Costa FIGUEIREDO² Elizandra Silva da PENHA ${ }^{2}$

Luanna Abílio Diniz Melquíades de MEDEIROS ${ }^{2}$

Gymenna Maria Tenório GUÊNES ${ }^{2}$

${ }^{1}$ Cirurgião(ã)-Dentista. Centro de Saúde e Tecnologia Rural, Universidade Federal de Campina Grande, UFCG 58708-110 Patos - PB, Brasil ${ }^{2}$ Professor(a) Doutor(a) do Curso de Graduação em Odontologia. Centro de Saúde e Tecnologia Rural,

Universidade Federal de Campina Grande, UFCG 58708-110 Patos - PB, Brasil

\section{Resumo}

Objetivou-se avaliar a influência que a estética dental apresenta na saúde do indivíduo. A amostra foi constituída por 150 pacientes de Odontologia, com idade acima 18 anos, de ambos os gêneros, realizada entre novembro de 2018 a março de 2019. Foram aplicados os questionários Sóciodemográfico, Impacto Psicossocial da Estética Dental e Escala da Autoestima de Rosenberg adaptado. Ao final, foram feitos os cálculos para obter os scores dos resultados para descobrir se há ou não impacto psicossocial da estética dental e se os pacientes possuíam baixa auto-estima. Destes 150 participantes, 103 (68,7\%) eram do gênero feminino; a maioria apresentou ter 39 ou mais anos de idade 48 (32\%); quanto à escolaridade resultaram em uma maioria de $39(26 \%)$ para os participantes que possuíam curso superior incompleto; acerca da renda per capita, a maioria $(98,65,4 \%)$ dos participantes do estudo relataram ter até um salário mínimo. Para o questionário de Impacto Psicossocial da Estética Dental, o score foi de 28,03 e no questionário de Autoestima, o score foi de 23,36. Portanto, a estética dentária teve grande impacto psicossocial na rotina dos pacientes e em virtude disso a autoestima se encontrava baixa segundo os resultados obtidos.

Descritores: Estética Dentária; Autoimagem; Saúde Bucal.

\section{Abstract}

The objective of this study was to evaluate the influence of dental aesthetics on the health of the individual. The sample consisted of 150 dentistry patients, aged 18 years and older, of both genders, performed between November 2018 and March 2019. The Socio-demographic, Psychosocial Impact of Dental Esthetics and Rosenberg Self-Esteem Scale were applied adapted. In the end, calculations were made to obtain the scores of the results to find out whether or not there is a psychosocial impact of dental aesthetics and whether the patients had low self-esteem. Of these 150 participants, $103(68.7 \%)$ were of the female gender; the majority presented having 39 or more years $48(32 \%)$; in terms of schooling resulted in a majority of 39 $(26 \%)$ for participants who had incomplete university degrees; about per capita income, the majority $(98,65.4 \%)$ of study participants reported having up to a minimum wage. For the Psychosocial Impact of Dental Esthetics questionnaire, the score was 28.03 and in the self-esteem questionnaire, the score was 23.36 . Therefore, dental aesthetics had a great psychosocial impact on the routine of patients and because of this self-esteem was low according to the results obtained.

Descriptors: Esthetics, Dental; Self Concept; Oral Health.

\section{Resumen}

El objetivo era evaluar la influencia que la estética dental tiene en la salud del individuo. La muestra consistió en 150 pacientes de odontología, mayores de 18 años, de ambos sexos, llevados a cabo entre noviembre de 2018 y marzo de 2019 . Se aplicaron el Impacto sociodemográfico, psicosocial de la estética dental y la Escala de autoestima adaptado de Rosenberg. Al final, se realizaron cálculos para obtener las puntuaciones de los resultados para determinar si existe o no un impacto psicosocial de la estética dental y si los pacientes tenían baja autoestima. De estos 150 participantes, 103 (68,7\%) eran mujeres; la mayoría presentó tener 39 o más años de edad 48 (32\%); en cuanto a la educación, una mayoría de 39 (26\%) resultó para participantes que tenían educación superior incompleta; con respecto al ingreso per cápita, la mayoría (98, 65.4\%) de los participantes del estudio informaron tener hasta un salario mínimo. Para el cuestionario Impacto psicosocial de la estética dental, el puntaje fue de 28.03 y en el cuestionario de autoestima, el puntaje fue de 23.36. Por lo tanto, la estética dental tuvo un gran impacto psicosocial en la rutina de los pacientes y, como resultado, la autoestima fue baja de acuerdo con los resultados obtenidos.

Descriptores: Estética Dental; Autoimagen; Salud Bucal.

INTRODUÇÃO

A boca é além de uma estrutura pertencente ao sistema digestório, um meio de se expressar, seja por meio da linguagem não verbal, por exemplo: um beijo, seja por meio da linguagem verbal através das palavras. Por isso, a manutenção da sua saúde, assim como de qualquer estrutura do corpo, se faz de extrema importância, visto que, é o principal meio de expressão do ser humano e, influencia diretamente em sua saúde ${ }^{1}$.

Estética é a apreciação da beleza ou a combinação de qualidades que proporcionam intenso prazer aos sentidos, aos conjuntos intelectuais e morais ${ }^{2}$. No geral, estética é a parte da filosofia voltada para a reflexão a respeito da beleza sensível e do fenômeno artístico. Os padrões de beleza estabelecidos pela cultura fazem com que a busca por tratamentos estéticos aumentem. É muito importante 0 encaixe das pessoas nesses padrões uma vez que, existem implicações psicológicas que influenciam a desenvoltura do indivíduo, acarretando assim na evolução ou involução do ser em quesitos como: relação 
interpessoal e autoestima, questões diretamente ligadas ao desenvolvimento do potencial humano e, por consequência, ao aumento ou diminuição da probabilidade de sucesso deste em áreas como: mercado de trabalho, relacionamentos interpessoais, etc ${ }^{3}$.

Dentre as estruturas da face, a boca juntamente com o sorriso constituem elementos fundamentais na estética facial. Apesar das diferentes percepções sobre estética facial entre pacientes e acadêmicos, ambos concordam com o fato de que os dentes são agentes imprescindíveis na harmonização facial ${ }^{4}$.

Atualmente, a busca pelo sorriso perfeito, tem mudado o enfoque da odontologia de tratamentos de doenças como cárie dentária para adequações estéticas do meio bucal à cultura. Tendo em vista a lei da oferta e da demanda, que visa à busca pela estabilização da procura de tratamento, é necessário que haja uma adaptação da oferta. Essa transição faz com que ocorra um abrupto alavancamento na área da estética dental ${ }^{1}$.

Atualmente, tanto em clínicas de universidades quanto em consultórios odontológicos, têm aumentado a procura de tratamento dentário por razões estéticas. Este fato pode fazer referência à maior demanda de informações através de revistas ou programas de televisão, que têm incentivado a procura por tratamentos de beleza e também pelo impacto pessoal no convívio social. Devido a essa informação crescente, muitos pacientes vêm se preocupando em harmonizar a relação dentogengival, procurando técnicas que esclareçam suas necessidades de um sorriso bonito ${ }^{5}$.

A crescente valorização da harmonização facial, incluindo um sorriso esteticamente agradável, faz com que os pacientes busquem cada vez mais alternativas de procedimentos estéticos ${ }^{6-8}$, recuperando forma e função, fisiologia mastigatória, harmonia e estética entre os arcos ${ }^{9}$.

Estando ciente da importância dos fatos supracitados, a estética dental faz-se relevante na manutenção da saúde bucal, tendo em vista que o paciente irá preservar os dentes com maior acuidade e, além disso, se mostra importante para o bem estar social do indivíduo. Logo, objetivou-se avaliar a relevância e a influência que a estética dental apresenta aos campos de saúde do indivíduo.

\section{MATERIAL E MÉTODO}

O desenho da pesquisa trata-se de um estudo transversal, com abordagem descritiva, utilizando um questionário específico para obtenção de dados no período de Novembro de
2018 a Março de 2019. O universo da pesquisa foi composto pelos pacientes em sala de espera na Clínica Escola de Odontologia da Universidade Federal de Campina Grande (UFCG), que se situa no município de Patos, localizada no Estado da Paraíba (PB) e a amostra foi composta por 150 (amostra obtida por conveniência) questionários aplicados e todos os participantes dela, todos assinaram o Termo de Assentimento e Termo de Consentimento Livre Esclarecido (TCLE).

A coleta de dados foi realizada pelo próprio pesquisador com o paciente na sala de espera aguardando atendimento em todas as especialidades da Clínica Escola. Para o auxílio na coleta dos dados, foram utilizados os questionários: sociodemográfico (idade, sexo, escolaridade), Impacto Psicossocial da Estética Dental que consiste em 23 itens que avaliam quatro dimensões da qualidade de vida relacionada à saúde bucal: autoconfiança dental, impacto social, impacto psicológico e aspectos estéticos, sendo que cada uma delas foi dado escores em uma escala do tipo Likert, valores de zero a quatro e no final foram somados a fim de se obter um escore final total que vai de 0 a 30 , sendo o 0 o melhor estado de estética dental e 30 o pior.

A autoestima foi avaliada através da Escala de Autoestima desenvolvida por Rosenberg $^{10}$ na versão adaptada para o português por Hutz e Zanon ${ }^{11}$, cujos resultados indicaram a unidimensionalidade do instrumento e características psicométricas equivalentes às encontradas originalmente. A medida é constituída por dez afirmações relacionadas a um conjunto de sentimentos de autoestima e autoaceitação que avalia a autoestima global. Os itens são respondidos em uma escala tipo Likert de quatro pontos variando entre concordo totalmente (4), concordo (3), discordo (2) e discordo totalmente (1). A pontuação total de autoestima compreende a soma dos itens e pode variar de 10 a 40 . Antes de obter a pontuação foi necessário inverter os itens contrários a autoestima, que correspondem aos itens 3, 5, 8, 9 e 10, Assim, a maior pontuação indica uma maior autoestima.

Tais instrumentos foram validados internacionalmente e traduzidos para a língua portuguesa com a finalidade de facilitação de aplicação e compreensão e se mostram disponíveis. Além disso, foi elaborado uma planilha eletrônica através do software Microsoft office excel com as informações obtidas através do questionário aplicado, e os dados coletados durante 0 decorrer da pesquisa foram tabelados. $O$ projeto foi submetido na 
plataforma brasil para apreciação do comitê de ética e aprovado sob o número do CAAE: 68482417.0.0000.5181.

\section{RESULTADOS}

\section{- Sóciodemográfico}

A amostra foi composta por 150 questionários aplicados nos pacientes na sala de espera da Clínica Escola de Odontologia da UFCG. Destes 150 participantes da pesquisa, $103(68,7 \%)$ eram do gênero feminino e 47 $(31,3 \%)$ eram do gênero masculino (Gráfico 1).

Quando questionados sobre a idade a maioria apresentou ter 39 ou mais anos 48 (32\%) (Gráfico 2). Os dados coletados referentes à escolaridade resultaram em uma maioria de 39 (26\%) para os participantes que possuíam curso superior incompleto (Tabela 1). Quando questionados acerca da renda per capita, a maioria $(98,65,4 \%)$ dos participantes do estudo relataram até um salário mínimo (Tabela 2).

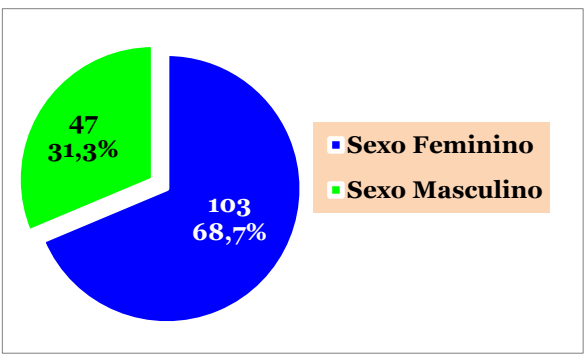

Gráfico 1: Dados referentes ao sexo.

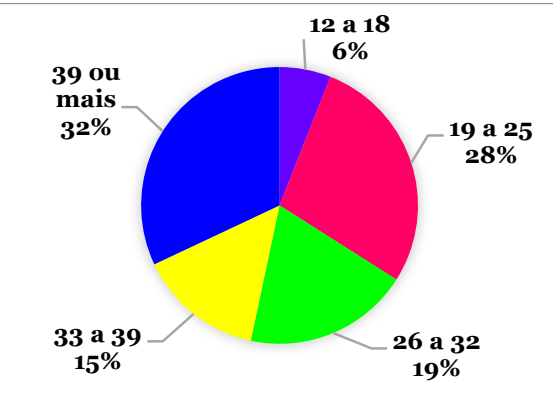

Gráfico 2: Dados referentes à faixa etária.

Tabela 1. Dados referentes à escolaridade

\begin{tabular}{|c|c|c|}
\hline \multicolumn{3}{|c|}{ ESCOLARIDADE } \\
\hline & No (Número) & \% (Por cento) \\
\hline NA & 1 & $0,7 \%$ \\
\hline EFI & 30 & $20 \%$ \\
\hline EFC & 8 & $5,3 \%$ \\
\hline EMI & 10 & $6,7 \%$ \\
\hline EMC & 38 & $25,3 \%$ \\
\hline CT & 9 & $6 \%$ \\
\hline CSI & 39 & $26 \%$ \\
\hline CSC & 15 & $10 \%$ \\
\hline TOTAL & 150 & $100 \%$ \\
\hline $\begin{array}{l}\text { NA: Não } \\
\text { incompleto; } \\
\text { Ensino médic } \\
\text { CT: Curso } \\
\text { CSC: Curso }\end{array}$ & $\begin{array}{l}\text { etizado; EFI: } \\
\text { Ensino Fundam } \\
\text { ompleto; EMC: En } \\
\text { ico; CSI: Curso } \\
\text { rior completo. }\end{array}$ & $\begin{array}{l}\text { Isino fundamental } \\
\text { tal Completo; EMl: } \\
\text { רo médio completo; } \\
\text { uperior incompleto; }\end{array}$ \\
\hline
\end{tabular}

Tabela 2. Dados referentes à renda

\begin{tabular}{lll}
\multicolumn{2}{c}{ RENDA PER CAPITA } \\
& $\mathbf{N}^{\mathbf{0}}$ (Número) & \% (Por cento) \\
\hline Até 1 salário mínimo & 98 & $65,4 \%$ \\
\hline 2 a 4 salários mínimos & 42 & $28 \%$ \\
\hline 5 a 6 salários mínimos & 8 & $5,3 \%$ \\
\hline 7 a 8 salários mínimos & 2 & $1,3 \%$ \\
\hline Mais de 9 salários mínimos & 0 & $0 \%$ \\
\hline TOTAL & 150 & $100 \%$ \\
\hline
\end{tabular}

- Impacto Psicossocial da Estética Dental

De acordo com o questionário de impacto psicossocial da estética dental, com as seguintes perguntas distribuídas em ítens: 1) Eu não gosto de ver meus dentes no espelho. 2) Eu seguro meu sorriso para não mostrar muito meus dentes. 3) Eu tenho inveja dos dentes bonitos de outras pessoas. 4) Eu tenho orgulho dos meus dentes. 5) Se eu não conheço bem uma pessoa eu me preocupo sobre o que ela pode estar pensando dos meus. 6) Eu me sinto um pouco aflito (a) quando vejo os dentes de outras pessoas. 7) Eu gosto de mostrar meus dentes quando eu sorrio. 8) Eu não gosto de ver meus dentes em fotografias. 9) Eu tenho medo de que as pessoas possam caçoar dos meus dentes. 10) Às vezes eu fico infeliz com a aparência dos meus dentes. 11) Eu acho que a maioria das pessoas que conheço tem dentes melhores que os meus. 12) Eu fico feliz quando eu vejo meus dentes no espelho. 13) Às vezes eu acho que as pessoas estão olhando para os meus dentes. 14) Eu fico um pouco inibido (a) em encontros sociais por causa dos meus dentes. 15) Às vezes me pego colocando minha mão em frente a minha boca para esconder meus dentes. 16) Sinto-me mal quando penso sobre como meus dentes se parecem. 17) Meus dentes são atrativos para os outros. 18) Eu não gosto de ver meus dentes quando vejo. 19) Comentários sobre meus dentes me irritam, mesmo quando são de brincadeira. 20) Eu gostaria que meus dentes fossem mais bonitos. 21) Eu estou satisfeito (a) com a aparência dos meus dentes. 22) Eu, às vezes, me preocupo com o que as pessoas do sexo oposto pensam sobre meus dentes. 23) Eu acho que meus dentes são muito bem posicionados. Os resultados obtidos estão descritos na Tabela 3 em forma de quantidade de assertivas respondidas e em porcentagem.

Tabela 3: Dados referentes aos impactos psicossociais da estética dental.

\begin{tabular}{llllll}
$\begin{array}{l}\text { Itens } \\
(\%)\end{array}$ & $\begin{array}{l}\text { De maneira } \\
\text { nenhuma }(\mathrm{o})\end{array}$ & $\begin{array}{l}\text { Um } \\
(1)\end{array}$ & $\begin{array}{l}\text { Mais } \\
\text { menos }(2)\end{array}$ & $\begin{array}{l}\text { Bastante } \\
(3)\end{array}$ & Muito (4) \\
\hline $\mathbf{1}$ & $62(41,3 \%)$ & $17(11,3 \%)$ & $37(24,6 \%)$ & $26(17,3 \%)$ & $8(5,3 \%)$ \\
\hline $\mathbf{2}$ & $84(56 \%)$ & $28(18,6)$ & $15(10 \%)$ & $13(8,6 \%)$ & $10(6,6 \%)$ \\
\hline $\mathbf{3}$ & $71(47,3 \%)$ & $32(21,3 \%)$ & $12(8 \%)$ & $19(12,7 \%)$ & $16(10,7 \%)$ \\
\hline $\mathbf{4}$ & $43(28,7 \%)$ & $18(12 \%)$ & $40(26,7 \%)$ & $32(21,3 \%)$ & $17(11,3 \%)$ \\
\hline $\mathbf{5}$ & $86(57,3 \%)$ & $24(16 \%)$ & $19(12,7 \%)$ & $17(11,3 \%)$ & $4(2,7 \%)$ \\
\hline $\mathbf{6}$ & $96(64 \%)$ & $18(12 \%)$ & $22(14,7 \%)$ & $9(6 \%)$ & $5(3 \%)$ \\
\hline $\mathbf{7}$ & $33(22 \%)$ & $22(14,7 \%)$ & $33(22 \%)$ & $30(20 \%)$ & $32(21,3 \%)$ \\
\hline $\mathbf{8}$ & $70(46,7 \%)$ & $27(18 \%)$ & $27(18 \%)$ & $20(13,3 \%)$ & $6(4 \%)$ \\
\hline $\mathbf{9}$ & $96(64 \%)$ & $20(13,3 \%)$ & $13(8,7 \%)$ & $13(8,7 \%)$ & $8(5,3 \%)$ \\
\hline $\mathbf{1 0}$ & $63(42 \%)$ & $33(22 \%)$ & $25(16,7 \%)$ & $21(14 \%)$ & $8(5,3 \%)$ \\
\hline $\mathbf{1 1}$ & $52(34,7 \%)$ & $21(14 \%)$ & $43(28,7 \%)$ & $25(16,7 \%)$ & $9(6 \%)$ \\
\hline $\mathbf{1 2}$ & $33(22 \%)$ & $18(12 \%)$ & $40(26,7 \%)$ & $38(25,3 \%)$ & $21(14 \%)$ \\
\hline $\mathbf{1 3}$ & $53(35,3 \%)$ & $33(22 \%)$ & $32(21,3 \%)$ & $28(18,7 \%)$ & $4(2,7 \%)$ \\
\hline $\mathbf{1 4}$ & $104(69,3 \%)$ & $18(12 \%)$ & $12(8 \%)$ & $13(8,7 \%)$ & $3(2 \%)$ \\
\hline $\mathbf{1 5}$ & $115(76,7 \%)$ & $9(6 \%)$ & $8(5,3 \%)$ & $11(7,3 \%)$ & $7(4,7 \%)$ \\
\hline $\mathbf{1 6}$ & $105(70 \%)$ & $20(13,3 \%)$ & $14(9,3 \%)$ & $7(4,7 \%)$ & $4(2,7 \%)$ \\
\hline $\mathbf{1 7}$ & $69(46 \%)$ & $22(14,7 \%)$ & $43(28,7 \%)$ & $11(7,3 \%)$ & $5(3,3 \%)$ \\
\hline $\mathbf{1 8}$ & $77(51,3 \%)$ & $30(20 \%)$ & $22(14,7 \%)$ & $16(10,7 \%)$ & $5(3,3 \%)$ \\
\hline $\mathbf{1 9}$ & $97(64,7 \%)$ & $18(12 \%)$ & $8(5,3 \%)$ & $18(12 \%)$ & $9(6 \%)$ \\
\hline $\mathbf{2 0}$ & $14(9,3 \%)$ & $23(15,3 \%)$ & $18(12 \%)$ & $32(21,3 \%)$ & $63(42 \%)$ \\
\hline $\mathbf{2 1}$ & $43(28,7 \%)$ & $31(20,7 \%)$ & $32(21,3 \%)$ & $27(18 \%)$ & $17(11,3 \%)$ \\
\hline $\mathbf{2 2}$ & $79(52,7 \%)$ & $28(18,7 \%)$ & $19(12,7 \%)$ & $15(9,3 \%)$ & $10(6,7 \%)$ \\
\hline $\mathbf{2 3}$ & $56(37,3 \%)$ & $17(11,3 \%)$ & $41(27,3 \%)$ & $20(13,3)$ & $16(10,7 \%)$ \\
\hline & & & & & \\
\hline
\end{tabular}


Obtidos os resultados individuais de cada item e de cada questionário, realizou-se 0 cálculo para obter a média segundo a metodologia, para o questionário de Impacto Psicossocial da Estética Dental, a score foi de 28,03 , isso significa que a estética tem grande impacto na dentição dos participantes, pois o valor se aproximou do score 30 , conforme a metodologia aplicada.

\section{- Autoestima}

Quanto ao questionário que avalia a autoestima dos participantes frente a sua saúde e estética bucal e geral, os dados foram expressos na Tabela 4 para melhor compreensão dos valores. Os itens analisados foram: 1) De uma forma geral (apesar de tudo), estou satisfeito (a) comigo mesmo. 2) Às vezes acho que não sirvo para nada (desqualificado (a) ou inferir em relação aos outros). 3) Eu sinto que tenho um tanto (um número) de qualidades. 4) Eu sou capaz de fazer coisas tão bem quanto a maioria das outras pessoas (desde de que ensinadas). 5) Não sinto satisfação nas coisas que realizei. Eu sinto que não tenho de que me orgulhar. 6) Às vezes, eu realmente me sinto inútil (incapaz de fazer as coisas). 7) Eu sinto que sou uma pessoa de valor, pelo menos num plano igual (num mesmo nível) às outras pessoas. 8) Não me dou o devido valor. Gostaria de ter mais respeito comigo mesmo (a). 9) Quase sempre estou inclinado a achar que sou uma pessoa fracassada. 10) Eu tenho atitude positiva (pensamentos, atos $\mathrm{e}$ sentimentos positivos).

Outrossim, no questionário de Autoestima proposto por Rosenberg ${ }^{10} \mathrm{e}$ adaptado por Hutz e Zanon ${ }^{11}$, a média foi de 23,36 , score esta que se aproxima mais do 10 que do 40, significando que houve baixa autoestima nos pacientes participantes da pesquisa.

Tabela 4. Dados referentes à autoestima

\begin{tabular}{|c|c|c|c|c|}
\hline \multicolumn{5}{|c|}{ QUESTIONÁRIO DE AUTO-ESTIMA } \\
\hline Itens (\%) & $\begin{array}{l}\text { Concordo } \\
\text { plenamente (o) }\end{array}$ & Concordo (1) & Discordo (2) & $\begin{array}{l}\text { Discordo } \\
\text { plenamente (3) }\end{array}$ \\
\hline $\mathbf{1}$ & $65(43,3 \%)$ & $65(43,3 \%)$ & $19(12,7 \%)$ & $1(0,7 \%)$ \\
\hline 2 & $3(2 \%)$ & $13(8,7 \%)$ & $54(36 \%)$ & $80(53,3 \%)$ \\
\hline 3 & $76(50,7 \%)$ & $66(44 \%)$ & $8(5,3 \%)$ & $\mathrm{o}(\mathrm{0} \%)$ \\
\hline 4 & $88(58,8 \%)$ & $56(37,3 \%)$ & $3(2 \%)$ & $3(2 \%)$ \\
\hline 5 & $2(1,3 \%)$ & $14(9,3 \%)$ & $52(34,7 \%)$ & $82(54,7 \%)$ \\
\hline 6 & $\mathrm{o}(\mathrm{0} \%)$ & $17(11,3 \%)$ & $52(34,7 \%)$ & $81(54 \%)$ \\
\hline 7 & $78(52 \%)$ & $68(43,3 \%)$ & $3(2 \%)$ & $1(0,7 \%)$ \\
\hline 8 & $10(6,7 \%)$ & $24(16 \%)$ & $52(34,7 \%)$ & $64(42,7 \%)$ \\
\hline 9 & $1(0,7 \%)$ & $10(6,7 \%)$ & $65(43,3 \%)$ & $74(49,3 \%)$ \\
\hline 10 & $84(56 \%)$ & $53(35,3 \%)$ & $12(8 \%)$ & $1(0,7 \%)$ \\
\hline
\end{tabular}

DISCUSSÃO

Neste estudo, foi possível perceber que - maior público da Clínica Escola de Odontologia que participou da pesquisa foi 0 feminino com 103 (68,7\%), corroborando com Flumingnan e Sampaio $\mathrm{Neto}^{12}$ que determinaram que o gênero feminino é predominância na procura do atendimento odontológico com $61,5 \%$. Tortamano et al. ${ }^{13}$ afirmam que a maior prevalência de mulheres pode ocorrer devido a uma maior preocupação com a saúde e cuidados estéticos. Reis et al. ${ }^{14}$ afirmaram que traçando um perfil dos pacientes atendidos em uma clínica integrada de odontologia, obteve o resultado de $67,8 \%$ dos pacientes atendidos sendo mulheres. Estudos realizados pelo ministério da saúde, afirmam os homens buscam menos por atendimento odontológico nos serviços de saúde, e o principal motivo seria o receio da descoberta de alguma doença considerada grave ${ }^{15}$.

Quanto à faixa etária, a maioria dos participantes da pesquisa possuía 39 anos acima (32\%). Concordando em partes com o que foi encontrado em nosso estudo, a pesquisa de Domingos et al. ${ }^{16}$ que trata do perfil sóciodemográfico dos pacientes atendidos em uma Clínica Escola de Odontologia, a faixa etária estava entre 41 e 50 anos (27,78\%).

Vigente até hoje, Rosemberg ${ }^{10}$ há muitos anos definiu autoestima como o sentimento ou apreço e a consideração que uma pessoa sente por si, ou seja, o quanto se gosta, como se vê e o que pensa sobre ela mesma. Centro da vida subjetiva do indivíduo, a autoestima determina os pensamentos, sentimentos e comportamentos do indivíduo.

Castilho ${ }^{17}$ destaca que os procedimentos estéticos são capazes de produzir aumento no bem estar psicológico do paciente por meio de mudanças na imagem corporal nos aspectos perceptivo, cognitivo, emocional e comportamental. Na Odontologia, a busca de procedimentos estéticos está fortemente atrelada ao desejo de melhorar a aparência e a autoestima, melhorando assim a qualidade de vida e o bem estar psicológico ${ }^{18-20}$.

Melo e Menezes Filho ${ }^{21}$ apontam que, a despeito do arsenal odontológico contar com eficientes ferramentas de análise do sorriso e referências estéticas, o sucesso do tratamento estético na Odontologia mantém estreita e vital relação com a correta interpretação das queixas estéticas dos pacientes. Mesquita ${ }^{22}$ acrescenta que um sorriso esteticamente agradável (dentes brancos dispostos harmonicamente) gera uma percepção positiva, o que possibilita ao indivíduo uma melhor aceitação e êxito no seu grupo social.

Nesta pesquisa, segundo o questionário aplicado de Impacto Psicossocial da Estética Dental foi possível perceber que os pacientes participantes da pesquisa encontravam-se abalados com sua estética dental, com uma média de 28,03 , seguindo a escala obtida pelo 
cálculo, que varia de 0 a 30 , sendo 30 o valor correspondente ao maior impacto negativo da estética dental.

Corroborando com esta pesquisa, em uma pesquisa feita por Gómez et al. $^{23} \mathrm{com}$ intuito de determinar o impacto psicossocial do estética dentária em estudantes com más oclusões e identificar o sexo e idade que parecem mais afetado. As maiores gravidades das más oclusões diminuem a autoestima dos indivíduos. O gênero mais afetado foi o feminino $59,1 \%$. A idade com o maior impacto foi de 16 anos. Quanto maior o grau de gravidade das más oclusões, maior impacto psicossocial, já que os alunos com má oclusão muito grave são aqueles eles mostraram baixa auto-estima em maior proporção.

Bem como em um estudo realizado por Franco et al. ${ }^{24}$ objetivando identificar as condições de saúde bucal de universitários do curso de odontologia de uma instituição de ensino particular e avaliar o impacto da saúde bucal na qualidade de vida dessa população, durante o período de 8 meses e com interesse em realizar clareamento dentário caseiro, os graduandos responderam a um questionário para mensurar o impacto da saúde bucal na qualidade de vida. Pôde-se concluir que, em um número expressivo, os universitários participantes se encontravam saudáveis e com a dentição hígida, entretanto, estavam insatisfeitos em relação à forma, posição e cor dos dentes. Não foi observado impacto negativo na qualidade de vida relacionada à saúde bucal.

Em um estudo realizado por Bersezio et al. ${ }^{25}$, também utilizando o questionário de impacto psicossocial da estética dental, sobre a Qualidade de vida e estabilidade da mudança de cor dos dentes aos três meses após o clareamento dental, com pacientes que fizeram clareamento em dente vital e não vital e avaliação da percepção estética e os fatores psicossociais avaliados 1 semana antes do tratamento e 1 mês após o clareamento, utilizando-se os questionários Oral Health Impact Profile (OHIP) e Impacto Psicossocial da Estética Dental, Bersezio verificou que ambos os agentes foram altamente eficazes e tiveram um impacto positivo na percepção estética e impacto psicossocial dos pacientes, resultados que também se mantiveram estáveis ao longo do tempo. O branqueamento não vital produziu impactos positivos e estáveis na percepção estética e fatores psicossociais.

A autoestima tem sido mensurada por meio da Escala de Autoestima de Rosenberg (EAR). Trata-se de um instrumento unidimensional capaz de classificar o nível de autoestima em baixo, médio e alto. A escala original foi desenvolvida para adolescentes e possui dez itens fechados, sendo cinco desses referentes à "autoimagem" ou "autovalor" positivos e cinco referentes à "autoimagem negativa" ou "autodepreciação". Os itens são dispostos no formato Likert de quatro pontos, variando entre "concordo totalmente" e "discordo totalmente" ${ }^{26,27}$. No Brasil, esse instrumento foi originalmente adaptado e validado para pesquisa por Hutz em 2000, cujos resultados indicaram a unidimensionalidade do instrumento e características psicométricas equivalentes às encontradas originalmente por Rosenberg ${ }^{11}$.

Neste estudo, utilizando o questionário específico de autoestima proposto por Rosenberg $^{10}$ e adaptado por Hutz e Zanon ${ }^{11}$, verificou-se que a autoestima dos pacientes participantes estava relativamente equilibrada, com uma média de 23,03, contudo, ainda mais próximo do valor 10 que corresponde à baixa autoestima, uma vez que, quanto mais próximo de 40 , segundo a metodologia proposta, melhor é a auto-estima do paciente.

Em um estudo feito por $\mathrm{Dini}^{28} \mathrm{com}$ objetivo testar a validade de construção e a sensibilidade "responsividade" da Escala de auto-estima de Rosenberg em 54 pacientes submetidos à lipoaspiração de ambos os sexos, com idade média de 28 anos, verificou-se aspectos emocionais, aspectos sociais, saúde mental, vitalidade, capacidade funcional, aspectos físicos e estado geral de saúde. Houve aumento das médias para os instrumentos: Escala de Autoestima Rosenberg. Apontou-se a melhora na qualidade de vida destes pacientes. A escala de autoestima de Rosenberg apresentou validade de construção e bons índices de sensibilidade "responsividade" em nosso meio.

Comparando a pesquisa de $\operatorname{Dini}^{28}$ com o nosso estudo, é possível perceber que procedimentos estéticos possuem influência positiva na qualidade de vida dos pacientes, uma vez que com o aumento das médias segundo a escala proposta adaptada, melhores são os resultados que os procedimentos estéticos promovem.

Por outro lado, adotando o público idoso, em uma pesquisa feita por Parentin Filho ${ }^{29} \mathrm{com}$ objetivo de avaliar a qualidade de vida e a autoestima em pacientes idosos que substituiram as próteses totais convencionais por overdentures mandibulares também utilizando a Escala de Autoestima de Rosenberg, verificou-se que não foram observadas diferenças estatísticas na Escala de 
AutoEstima Rosenberg, logo, houve impacto positivo na qualidade de vida, exceto da autoestima de idosos que substituíram as próteses totais convencionais por overdentures mandibulares.

Também utilizando a Escala de Autoestima Global, da autoria de Rosenberg, Com objetivo de relacionar a autoestima dos indivíduos com o uso de prótese dentária removível total e/ou parcial, para avaliar se o uso de prótese dentária removível leva a variações no grau de autoestima dos pacientes parcialmente e totalmente desdentados, nomeadamente a uma baixa autoestima e se há diferenças de autoestima consoante o tempo de uso da prótese e consoante o tipo de prótese usada, tendo em conta indicadores socioeconômicos e culturais, idade e gênero, Carvalho $^{30}$ concluiu que os valores de autoestima obtidos foram satisfatórios visto que os inquiridos apresentam uma pontuação média de autoestima de 51,58 pontos, (mínimo de 10 pontos e um máximo de 60 pontos) adaptada para o caso. Este valor corresponde a uma autoestima elevada. Logo, Independentemente do género, idade, nível socioeconômico e cultural, tipo de prótese usada e tempo de uso de prótese a autoestima dos participantes deste estudo parece ser elevada.

Concordando com isso, em um estudo realizado por Santos et al. ${ }^{31}$ com objetivo de avaliar o impacto da imagem corporal e autoestima, também utilizando em sua metodologia, dentre outras formas de coleta de dados, a Escala de Autoestima de Rosenberg, obteve que dos 719 estudantes universitários, de ambos os sexos, com idade entre 18 a 59 anos, matriculados nos dez cursos de graduação da Universidade Federal de Viçosa observou-se que as mulheres apresentaram maior preocupação com a imagem corporal e menor autoestima em relação aos homens.

Em contra partida, um estudo feito por Gomes $^{32}$ com objetivo de avaliar as relações entre nível socioeconômico, apoio social, fatores psicossociais, comportamentos em saúde e qualidade de vida relacionada à saúde em adolescentes. Um estudo de seguimento de 6 meses de base escolar envolvendo 376 adolescentes com 12 anos de idade foi realizado em Manaus, Brasil. A coleta de dados foi realizada através de questionários autoaplicáveis para os adolescentes e seus responsáveis. Dentre muitos dados relacionados como objetivo da pesquisa, um dos resultados dele foi maior autoestima $(\beta=0.23)$, logo, tendo estes adolescentes boa autoestima. Além disso, níveis socioeconômicas, apoio social e fatores psicossociais foram preditores importantes para comportamentos em saúde e Qualidade de Vida Relacionada a Saúde de adolescentes através de mecanismos diretos e indiretos. Comportamentos em saúde influenciaram diretamente a Qualidade de Vida Relacionada à Saúde.

A autoestima ou a avaliação geral positiva que cada um faz de si mesmo associase com mais satisfação com a vida e menos problemas de saúde ${ }^{33}$. A influência da vida social e da autopercepção são fatores importantes, que têm um papel essencial na autoestima de uma pessoa em relação à má oclusão. Alguns pacientes com grave má oclusão estão satisfeitos ou indiferentes com sua estética, enquanto outros, com irregularidades menores, preocupam-se muito com este quesito. Ou seja, a necessidade normativa e subjetiva do indivíduo pode variar quando se trata de tratamentos ortodônticos ${ }^{34}$.

CONCLUSÃO

Portanto, a estética dentária teve grande impacto psicossocial na rotina dos pacientes atendidos na clínica escola de odontologia da UFCG observada no questionário de Impacto Psicossocial da Estética Dental, e em virtude disso a autoestima se encontrava baixa conforme os resultados do questionário de Rosenberg validado e adaptado, evidenciando a insatisfação que os pacientes participantes da pesquisa tem com sua autoimagem e autoaceitação.

\section{REFERÊNCIAS}

1. Gaikwad S, Kaur H, Vaz AC, et al. Influence of Smile Arc and Buccal Corridors on Facial Attractiveness: A Cross-sectional Study. J Clin Diagn Res. 2016;10(9):ZC20-ZC23.

2. Okida RC, Vieira WSC, Rahal V, Okida DSS. Lentes de contato: restaurações minimamente invasivas na solução de problemas estéticos. Rev Odontol Araçatuba. 2016;37(1):53-9.

3. Borges CM, Peres MA, Peres KG. Associação entre presença de oclusopatias e insatisfação com a aparência dos dentes e gengivas: estudo com adolescentes brasileiros. Rev bras epidemiol. 2010;13(4):713-23.

4. Feitosa DAS, Dantas ACRE, Guênes GMT, Ribeiro AIAM, Cavalcanti AL, Braz R. Percepção de pacientes e acadêmicos de odontologia sobre estética facial e dentária. RFO UPF. 2009;14(1):23-6.

5. Sousa CP, Garzon ACM, Sampaio JEC. Estética periodontal: relato de um caso. Rev Bras Ci Period. 2003;1:262-67.

6. Cardenas AFM, Mora CAP, Siqueira FSF, Parreiras SO, Gomes JC. Restabelecimento 
estético de um sorriso envelhecido: Caso clínico. Revista APCD Estética. 2015;3(1): 42-52.

7. Strasding M, Fehmer V, Pjetursson BE, Sailer I. Extending the service life of existing dental restorations with esthetic and functional limitations. J Prosthet Dent. 2018;119(6): 893-96.

8. Koidou VP, Rosenstiel SF, Rashid RG. Celebrity smile esthetics assessment: Smile angulation. J Prosthet Dent. 2017;117(5): 636-41.

9. Cruz JHA, Silva RLB, Andrade Junior FP, Guênes GMT, Almeida MSC, Medeiros LADM et al. A importância da anatomia e escultura dental para prática de procedimentos clínicos odontológicos. RSC online. 2018;7(1):76-85.

10. Rosenberg M. Society and the adolescent selfimage. Revised edition. Middletown, CT: Wesleyan University Press; 1989.

11. Hutz CS, Zanon C. Revisão da adaptação, validação e normatização da Escala de Autoestima de Rosenberg. Aval psicol. 2011;10(1):41-9.

12. Flumignan JDP, Sampaio Neto LF. Atendimento odontológico em unidades de emergência: caracterização da demanda. Rev Bras Odontol. 2014;71(2):124-29.

13. Tortomano IP, Leopoldino VD, Borsatti MA, Sarti Penha S, Buscariolo IA, Costa CG et al. Aspectos Epidemiológicos e Sociodemográficos do setor de urgência da Faculdade de Odontologia da Universidade de São Paulo. RPG Rev Pós Grad. 2007;13(14):299-306.

14. Reis S, Santos L, Leles C. Clínica Integrada de Ensino Odontológico: Perfil dos usuários e necessidades odontológicas. ROBRAC. 2011; 20(52):46-51.

15. Gomes R, Nascimento EF, Araújo FC. Por que os homens buscam menos os serviços de saúde do que as mulheres? As explicações de homens com baixa escolaridade e homens com ensino superior. Cad Saúde Pública. 2007;23(3):565-74.

16. Domingos $P$, Rossato $E$, Bellini $A$. Levantamento do perfil social, demográfico econômico de pacientes atendidos na clínica de Odontologia do centro universitário da Araraquara. Rev UNIARA. 2014;17(1):37-50.

17. Castilho SM. A imagem corporal. Santo André: Esetec; 2001.

18. Carminatti M, Fajardo RS, Alves Rezende MCR. Humanização do atendimento em saúde: perfil e expectativas de egressos de odontologia. Arch Health Invest 2013;2 (Especial 2 ):134.

19. Capalbo LC, Carminatti M, Capalbo BC, Cury MT, Fiorin LG, Wada CM et al. Atendimento humanizado: perfil e expectativas de odontolandos. Arch Health Invest. 2014;3:(Spec Iss 3):15-6.

20. Guerra CT, Alves Rezende MCR. Humanização do atendimento em saúde: perfil dos cirurgiõesdentistas [ monografia]. Araçatuba: Faculdade de Odontologia de Araçatuba, Universidade Estadual Paulista, Araçatuba; 2014.

21. Melo GFB, Menezes Filho PF. Proporção áurea e sua relevância para a odontologia estética. Int J Dent. 2008;7(4):234-38.

22. Mesquita MS. O sorriso humano [dissertação]. Lisboa: Universidade de Lisboa; 2011.

23. Gómez EL, San Martín AL, García MJ, García A, Mendoza LV, San Martín A. Impacto psicosocial de la estética dental en alumnos con maloclusiones del Telebachillerato Coxquihui, Veracruz. Rev Mex Med Forense, 2019, 4(suppl 1):54-7

24. Franco TPM, Motta VC, Canabarro A, Tannure PN. Perfil dos universitários de odontologia e o impacto da saúde bucal na qualidade de vida dessa população. Rev Odontol Univ Cid São Paulo. 2018;30(3):256-64.

25. Bersezio C, Martín J, Mayer C, Rivera O, Estay J, Vernal R, Haidar ZS, Angel P, Oliveira OB Jr, Fernández E. Quality of life and stability of tooth color change at three months after dental bleaching. Qual Life Res. 2018;27(12):31993207.

26. Sbicigo JB, Bandeira DR, Dell'aglio DD. Escala de Autoestima de Rosenberg (EAR): validade fatorial e consistência interna. Psico-USF. 2010;15(3):395-403.

27. Avanci JQ, Assis SG, Santos NC, Oliveira RVC. Adaptação transcultural de escala de autoestima para adolescentes. Psicol Reflex Crit 2007;20:397-405.

28. Dini GM. validade de construção e sensibilidade da escala de auto-estima Rosenberg/Unifesp-EPM em lipoaspiração [tese]. São Paulo: Universidade Federal de São Paulo - Escola Paulista de Medicina; 2004.

29. Parenti Filho A. Short form-36, escala de autoestima rosenberg-unifesp/epm, oral health impact profile em idosos com overdentures mandibulares [dissertação]. São Paulo: Universidade Federal de Sao Paulo; 2010.

30. Carvalho MFT. Avaliação da auto-estima nos portadores de Prótese Dentária Removível [monografia] Porto: Universidade Fernando Pessoa - Faculdade de Ciências da Saúde; 2009.

31. Santos VS, Patto MV, Cornélio MPM, Carleto CT, Pedrosa LAK. Preocupação com a Imagem Corporal e a Autoestima de Universitários do Interior de Minas Gerais. Rev Bras Educ Cult. 2019;19:95-105.

32. Gomes, AC. Condição socioeconômica, apoio social, fatores psicossociais, comportamentos em saúde e qualidade de vida relacionada à 
saúde em adolescentes: Um estudo de seguimento de 6 meses [dissertação]. Manaus: Programa de Pós-Graduação da Faculdade de Odontologia da Universidade Federal do Amazonas; 2019.

33. Agou S, Locker D, Streiner DL, Tompson B. Impact of self-esteem on the oral-health-related quality of life of children with malocclusion. Am J Orthod Dentofacial Orthop. 2008;134(4): 484-89.

34. Sharmaa A, Mathura A, Batraa M, Makkara DK, Aggarwala VP, Goyala N et al. Avaliação objetiva e subjetiva da necessidade de tratamento ortodôntico do adolescente e seu impacto sobre a autoestima. Rev Paul Pediatr. 2017;35(1):86-91.

\section{CONFLITO DE INTERESSES}

Os autores declaram não haver conflitos de interesse

AUTOR PARA CORRESPONDÊNCIA

\section{José Henrique de Araújo Cruz}

Rua Paulo Diogenes 57 - Centro

59990000 Rafael Fernandes - RN, Brasil

Telefone: (83) 99625-0125.

E-mail: henrique_araujo1992@hotmail.com

Submetido em 03/04/2020

Aceito em 23/10/2020 